\title{
BMI, waist circumference, and clustering of cardiovascular risk factors in Japanese adults
}

\author{
Machi Suka $\cdot$ Yuichi Miwa Yoshiki Ono $\cdot$ \\ Hiroyuki Yanagisawa
}

Received: 5 February 2010/ Accepted: 14 July 2010/Published online: 11 August 2010

(C) The Japanese Society for Hygiene 2010

\begin{abstract}
Objective To examine whether the association between waist circumference (WC) and clustering of cardiovascular risk factors varies with obesity (BMI) status.

Methods Using the 2008 health examination data of a Japanese health service association, eligible 57,141 adults aged 20-65 years without coronary heart disease or stroke, whose blood sample had been taken in the fasting state, were enrolled in the study. The participants were classified as being underweight (BMI $<18.5$ ), normal weight (BMI 18.5-24.9), and overweight (BMI $\geq 25.0$ ). Multiple logistic regression analysis was performed to calculate adjusted odds ratios (OR) for clustering of cardiovascular risk factors. Receiver operating characteristic analysis was performed to assess the ability of WC to discriminate subjects with and without a clustering of cardiovascular risk factors. Results Clustering of cardiovascular risk factors was found in $16.0 \%$ of men and $3.4 \%$ of women. The adjusted OR [95\% confidence intervals (CI)] per 5-cm increase in WC of the underweight, normal weight, and overweight groups was 1.57 (1.12-2.20), 1.55 (1.49-1.62), and 1.34 (1.30-1.38), respectively, for men and $1.50(0.84-2.69)$, $1.53(1.40-1.68)$, and $1.32(1.23-1.41)$, respectively, for women. The area under curve $(95 \% \mathrm{CI})$ of the underweight, normal weight, and overweight groups was 0.68 (0.59-0.77), $0.70 \quad(0.69-0.71)$, and 0.62 (0.61-0.63),
\end{abstract}

\footnotetext{
M. Suka $(\bowtie) \cdot H$. Yanagisawa

Department of Public Health and Environmental Medicine, Jikei University School of Medicine, 3-25-8 Nishi-Shimbashi, Minato-ku, Tokyo 105-8461, Japan

e-mail: suka@jikei.ac.jp

Y. Miwa $\cdot$ Y. Ono

Tokyo Health Service Association, Tokyo, Japan
}

respectively, for men and $0.70(0.53-0.86), 0.75(0.73-0.78)$, and 0.64 (0.61-0.68), respectively, for women.

Conclusion High WC was associated with increased risk of clustering of cardiovascular risk factors independent of BMI. As well as the magnitude of the association, the ability of WC to discriminate subjects with and without a clustering of cardiovascular risk factors varied with obesity (BMI) status.

Keywords Waist circumference - Body mass index . Cardiovascular risk factors - Metabolic syndrome - Japan

\section{Introduction}

The metabolic syndrome is characterized by a clustering of cardiovascular risk factors in one person, and those individuals with the metabolic syndrome are at increased risk of developing coronary heart disease and other arteriosclerotic diseases. The metabolic syndrome has become increasingly common in Japan as well as in Western countries. Based on the results of the 2008 National Health and Nutrition Survey, an estimated $25.3 \%$ of men and $10.6 \%$ of women aged $\geq 20$ years have the metabolic syndrome [1]. The Japanese Ministry of Health, Labour, and Welfare initiated a nationwide strategy for the detection of metabolic syndrome in April 2008 [2]. The measurement of waist circumference (WC) is a key component of this strategy, and the measurement results are used as a diagnostic indicator of the metabolic syndrome [3].

$\mathrm{WC}$ is a surrogate marker of visceral fat mass. Body mass index (BMI) is more widely used in clinical settings, but it does not provide information on fat distribution. Many investigators have reported an association between WC and clustering of cardiovascular risk factors and the 
optimal WC cutoff for the detection of clustering of cardiovascular risk factors in Japanese adults [4-13]. However, because of a strong positive correlation between BMI and WC, it appears to be difficult to completely discriminate the effects of BMI and WC, respectively, on clustering of cardiovascular risk factors. Unfortunately, there have been few studies that have assessed the association between WC and clustering of cardiovascular risk factors in terms of potential BMI dependence. It remains uncertain whether WC has an adequate ability to discriminate subjects with and without a clustering of cardiovascular risk factors within all BMI categories.

The objective of this study was to examine whether the association between WC and clustering of cardiovascular risk factors varies with obesity (BMI) status. Using the 2008 health examination data of a Japanese health service association, we compared the magnitude of the association between WC and clustering of cardiovascular risk factors and the ability of WC to discriminate subjects with and without a clustering of cardiovascular risk factors across BMI categories.

\section{Methods}

Annual multiphasic health examinations are legally mandatory in both the community and workplace setting in Japan. Electronic data of the 2008 health examinations were collected from the Tokyo Health Service Association, where a total 160,000 adults undergo a health examination every year (http://www.yobouigaku-tokyo.or.jp). Each record consisted of demographics and the results of laboratory tests and imaging tests. Eligible 57,141 adults aged 20-65 years without coronary heart disease or stroke, whose blood sample had been taken in the fasting state, were included enrolled in this study. The mean age was 44.2 [standard deviation (SD) 10.7] years for men $(n=37,792)$ and 42.9 (11.1) years for women $(n=19,349)$.

Anthropometric measurements and laboratory assays were performed according to a standard protocol. Height (in centimeters to the nearest $0.1 \mathrm{~cm}$ ) and weight (in kilograms to the nearest $0.1 \mathrm{~kg}$ ) were measured with a subject light clothed and standing without shoes. WC (in centimeters to the nearest $0.5 \mathrm{~cm}$ ) was measured horizontally at the umbilical level at the end of normal expiration by trained nurses. Blood pressure was measured using a standard sphygmomanometer with the subject sitting on a chair after at least 5 min of rest. Blood glucose, triglycerides, and high-density lipoprotein (HDL) cholesterol were assayed at a laboratory of the Tokyo Health Service Association, where both internal and external quality controls of the laboratory data are regularly in accordance with the guidelines of the expert committee for data standardization [14].

BMI, which was calculated as weight (kilograms) divided by height squared (square meters), was classified as $<18.5$ (underweight), 18.5-24.9 (normal weight), and $\geq 25.0$ (overweight). Cardiovascular risk factors were defined according to the criteria for metabolic syndrome in Japan [3]. Subjects who had any two of the following three components of metabolic syndrome were counted as a case with clustering of cardiovascular risk factors: (1) hypertension: systolic blood pressure $\geq 130 \mathrm{mmHg}$ and/or diastolic blood pressure $\geq 85 \mathrm{mmHg}$; (2) hyperglycemia: fasting blood glucose $\geq 110 \mathrm{mg} / \mathrm{dl}$ ( $5.6 \mathrm{mmol} / \mathrm{l}$ ); (3) dyslipidemia: triglyceride $\geq 150 \mathrm{mg} / \mathrm{dl}(1.7 \mathrm{mmol} / \mathrm{l})$ and/or HDL cholesterol $<40 \mathrm{mg} / \mathrm{dl}(1.0 \mathrm{mmol} / \mathrm{l})$. Subjects on medication for hypertension, diabetes mellitus, or hypertriglyceridemia were classified as having these cardiovascular risk factors. This definition of clustering of cardiovascular risk factors has also been used in recent epidemiological studies [5-7, 9-13].

The study protocol was approved by the Ethics Committee of the Tokyo Health Service Association and has been conducted in accordance with the Guidelines for Epidemiological Studies by the Japanese Ministry of Health, Labour, and Welfare and the Japanese Ministry of Education, Culture, Sports, Science, and Technology.

All statistical analyses were performed using the Statistical Analysis Systems (SAS, ver. 9.2; SAS Institute, Cary, NC). Analysis of variance (ANOVA) was used to test for statistical differences among the mean values. The chisquare test was used to test for statistical differences among the prevalence values. A multiple logistic regression model was developed in each BMI group to assess the association between WC and clustering of cardiovascular risk factors. Adjusted odds ratio (OR) with $95 \%$ confidence intervals (CI) for clustering of cardiovascular risk factors was calculated per 5-cm increase in WC with adjustment for age. Moreover, a receiver operating characteristic (ROC) curve was created in each BMI group to assess the ability of WC to discriminate subjects with and without a clustering of cardiovascular risk factors. The area under curve (AUC) was computed with the PLOC LOGISTIC procedure of the SAS program. The most discriminative threshold with the maximal sensitivity plus specificity was determined as the optimal WC cutoff for the detection of clustering of cardiovascular risk factors. The sensitivity and specificity of the BMI-specific WC cutoffs, compared with the single WC cutoffs recommended by the International Diabetes Federation (IDF) $(90 \mathrm{~cm}$ for men and $80 \mathrm{~cm}$ for women) [15] and by the Japanese Committee $(85 \mathrm{~cm}$ for men and $90 \mathrm{~cm}$ for women) [3], were calculated in each BMI group. 
Table 1 Characteristics of study subjects

\begin{tabular}{|c|c|c|c|c|c|c|c|c|}
\hline & \multicolumn{4}{|l|}{ Men } & \multicolumn{4}{|l|}{ Women } \\
\hline & \multirow{2}{*}{$\begin{array}{l}\text { All } \\
(n=37,792)\end{array}$} & \multicolumn{3}{|l|}{ BMI } & \multirow{2}{*}{$\begin{array}{l}\text { All } \\
(n=19,349)\end{array}$} & \multicolumn{3}{|l|}{ BMI } \\
\hline & & $\begin{array}{l}\text { Underweight } \\
(n=1,243)\end{array}$ & $\begin{array}{l}\text { Normal } \\
\text { weight } \\
(n=25,680)\end{array}$ & $\begin{array}{l}\text { Overweight } \\
(n=10,869)\end{array}$ & & $\begin{array}{l}\text { Underweight } \\
(n=3,124)\end{array}$ & $\begin{array}{l}\text { Normal } \\
\text { weight } \\
(n=13,810)\end{array}$ & $\begin{array}{l}\text { Overweight } \\
(n=2,415)\end{array}$ \\
\hline \multicolumn{9}{|c|}{ Anthropometric variables (mean \pm standard deviation) } \\
\hline BMI & $23.6 \pm 3.3$ & $17.6 \pm 0.7$ & $22.2 \pm 1.7$ & $27.5 \pm 2.6^{*}$ & $21.4 \pm 3.4$ & $17.5 \pm 0.8$ & $21.1 \pm 1.7$ & $28.0 \pm 3.0^{*}$ \\
\hline $\begin{array}{l}\text { Waist circumference } \\
(\mathrm{cm})\end{array}$ & $83.6 \pm 8.8$ & $68.8 \pm 4.0$ & $80.3 \pm 5.7$ & $93.0 \pm 7.3^{*}$ & $76.5 \pm 9.3$ & $67.1 \pm 4.6$ & $75.9 \pm 6.5$ & $91.7 \pm 8.2^{*}$ \\
\hline \multicolumn{9}{|c|}{ Metabolic variables (mean \pm standard deviation) } \\
\hline $\begin{array}{l}\text { Systolic blood pressure } \\
(\mathrm{mmHg})\end{array}$ & $120.4 \pm 14.9$ & $112.1 \pm 14.3$ & $118.3 \pm 14.2$ & $126.4 \pm 14.7^{*}$ & $111.6 \pm 15.3$ & $105.3 \pm 13.1$ & $110.9 \pm 14.5$ & $123.9 \pm 15.6^{*}$ \\
\hline $\begin{array}{l}\text { Diastolic blood pressure } \\
(\mathrm{mmHg})\end{array}$ & $75.2 \pm 11.6$ & $68.5 \pm 10.8$ & $73.6 \pm 11.2$ & $79.8 \pm 11.3 *$ & $67.7 \pm 10.9$ & $63.9 \pm 9.6$ & $67.2 \pm 10.4$ & $75.5 \pm 11.0^{*}$ \\
\hline $\begin{array}{l}\text { Fasting blood glucose } \\
(\mathrm{mg} / \mathrm{dl})\end{array}$ & $95.7 \pm 20.1$ & $89.6 \pm 15.9$ & $93.9 \pm 17.2$ & $100.6 \pm 25.4^{*}$ & $88.3 \pm 11.9$ & $85.6 \pm 10.4$ & $87.8 \pm 10.4$ & $95.0 \pm 18.1^{*}$ \\
\hline $\begin{array}{l}\text { Triglyceride } \\
(\mathrm{mg} / \mathrm{dl})\end{array}$ & $126.3 \pm 103.6$ & $78.1 \pm 71.6$ & $111.8 \pm 86.4$ & $166.2 \pm 129.4^{*}$ & $74.0 \pm 48.7$ & $59.4 \pm 48.0$ & $71.2 \pm 41.9$ & $108.9 \pm 66.6^{*}$ \\
\hline $\begin{array}{l}\text { HDL cholesterol } \\
(\mathrm{mg} / \mathrm{dl})\end{array}$ & $58.8 \pm 14.0$ & $68.4 \pm 15.4$ & $60.8 \pm 14.1$ & $52.9 \pm 11.6^{*}$ & $71.3 \pm 14.8$ & $76.7 \pm 14.6$ & $71.7 \pm 14.3$ & $61.8 \pm 13.1 *$ \\
\hline \multicolumn{9}{|l|}{ Prevalence $(\%)$} \\
\hline Hypertension & 29.9 & 12.7 & 23.7 & $46.4^{*}$ & 14.0 & 5.4 & 11.8 & $37.5^{*}$ \\
\hline Hyperglycemia & 9.9 & 3.3 & 7.4 & $16.7 *$ & 3.0 & 1.0 & 2.2 & $9.9 *$ \\
\hline Dyslipidemia & 27.2 & 5.9 & 20.4 & $45.8^{*}$ & 5.9 & 1.7 & 4.7 & $18.4^{*}$ \\
\hline $\begin{array}{l}\text { Clustering of } \\
\text { cardiovascular } \\
\text { risk factors }\end{array}$ & 16.3 & 3.3 & 10.3 & $31.0^{*}$ & 3.4 & 0.3 & 2.1 & $14.8 *$ \\
\hline
\end{tabular}

$B M I$ body mass index, $H D L$ high-density lipoprotein

$* p<0.001$ among BMI groups

\section{Results}

Table 1 shows the characteristics of the study subjects. For the 37,792 men, mean BMI was 23.6 (SD 3.3), and the underweight and overweight groups accounted for 3.3 and $28.8 \%$ male study population, respectively. For the 19,349 women, mean BMI was 21.4 (3.4), and the underweight and overweight groups accounted for 16.1 and $12.5 \%$ of the female study population, respectively. The mean WC of the underweight, normal weight, and overweight groups showed an approximately $10-\mathrm{cm}$ difference between the groups for both sexes $(p<0.001)$. Hypertension, hyperglycemia, dyslipidemia, and clustering of cardiovascular risk factors were found in 29.9, 9.9, 27.2, and $16.0 \%$, respectively, of men and 14.0, 3.0, 5.9, and 3.4\%, respectively, of women, with significant BMI-dependent increa$\operatorname{ses}(p<0.001)$.

Table 2 shows the adjusted OR per 5-cm increase in WC for clustering of cardiovascular risk factors. The adjusted OR per $5-\mathrm{cm}$ increase in WC for clustering of cardiovascular risk factors was significantly $>1.0$ in all BMI groups,
Table 2 Adjusted odds ratios per 5-cm increase in waist circumference for clustering of cardiovascular risk factors

\begin{tabular}{lll}
\hline BMI & \multicolumn{2}{l}{ Odds ratio $(95 \%$ confidence interval) } \\
\cline { 2 - 3 } & Men & Women \\
\hline All & $1.36(1.33-1.40)^{\mathrm{a}}$ & $1.26(1.18-1.35)^{\mathrm{a}}$ \\
Underweight & $1.57(1.12-2.20)^{\mathrm{b}}$ & $1.50(0.84-2.69)^{\mathrm{b}}$ \\
Normal weight & $1.55(1.49-1.62)^{\mathrm{b}}$ & $1.53(1.40-1.68)^{\mathrm{b}}$ \\
Overweight & $1.34(1.30-1.38)^{\mathrm{b}}$ & $1.32(1.23-1.41)^{\mathrm{b}}$ \\
\hline
\end{tabular}

${ }^{a}$ Adjusted for age and BMI

b Adjusted for age

with the exception of the underweight group of women, in which the number of subjects with a clustering of cardiovascular risk factors was very few. Compared with the normal weight group, the overweight group had a significantly smaller OR value, while the underweight group had an almost equivalent $\mathrm{OR}$ value.

Figure 1 shows the ROC curves of WC for clustering of cardiovascular risk factors. Compared with the normal 

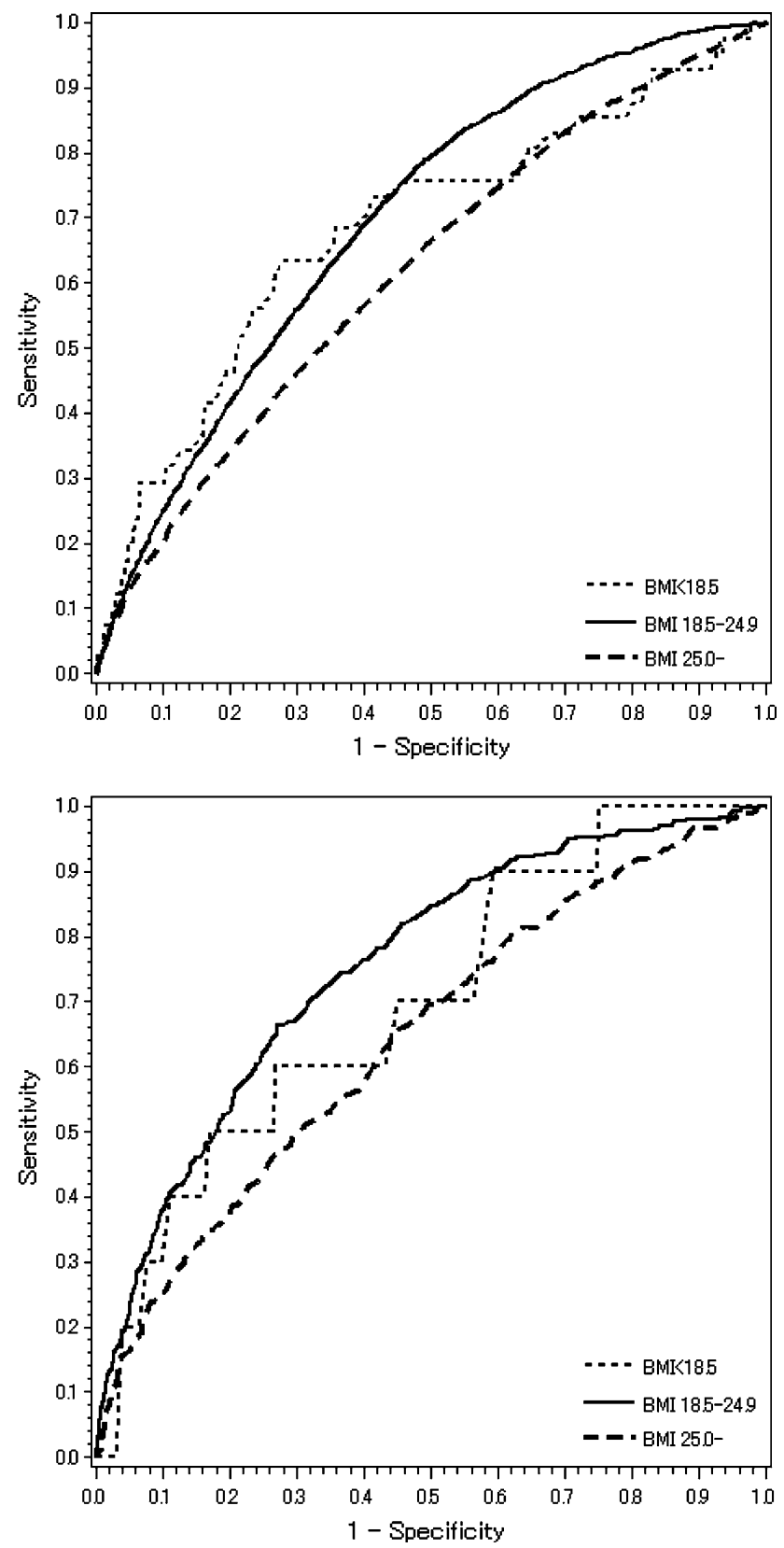

Fig. 1 Receiver operating characteristic (ROC) curves of waist circumference for clustering of cardiovascular risk factors in men (upper) and women (lower). BMI body mass index

weight group, the overweight group had a curve closer to the $45^{\circ}$ diagonal of the ROC space. The underweight group lay midway between the other two groups. The AUC $(95 \%$ CI) of the underweight, normal weight, and overweight groups was 0.68 (0.59-0.77), $0.70(0.69-0.71)$, and 0.62 (0.61-0.63), respectively, for men and $0.70(0.53-0.86)$, $0.75(0.73-0.78)$, and $0.64(0.61-0.68)$, respectively, for women. In all BMI groups combined, the maximal sensitivity plus specificity was achieved at a WC of $85 \mathrm{~cm}$ for men and $81 \mathrm{~cm}$ for women. The most discriminative threshold of WC of the underweight, normal weight, and overweight groups was estimated to be 69,81 , and $92 \mathrm{~cm}$, respectively, for men and 67,78 , and $91 \mathrm{~cm}$, respectively, for women.

Table 3 shows the sensitivity and specificity of single and BMI-specific WC cutoffs used to discriminate subjects with and without a clustering of cardiovascular risk factors. When the single WC cutoffs recommended by the IDF [15] and by the Japanese Committee [3] were applied to the study subjects, the prevalence of a high WC (percentage of subjects with a WC above the cutoff) differed widely between the BMI groups. The sensitivity was considerably lower in the underweight and normal weight groups, while the specificity was considerably lower in the overweight group. When the BMI-specific WC cutoffs were applied to the study subjects, the prevalence of high WC was around $50 \%$ in all BMI groups. For both sexes, the sensitivity in underweight and normal weight groups exceeded 0.7 and the specificity in the overweight group exceeded 0.5.

The discriminatory ability of BMI-specific WC cutoffs was compared with that of the single WC cutoff obtained from this study. When all of the study subjects were taken into account, the sensitivity and specificity of BMI-specific WC cutoffs was estimated to be 0.67 and 0.55 , respectively, for men and 0.70 and 0.60 , respectively, for women, which were rather lower than those of the single WC cutoff. When the study subjects were divided into those without excess weight (the combined underweight and normal weight group) and those with excess weight (the overweight group), the BMI-specific WC cutoffs provided a substantial improvement in sensitivity in those subjects without excess weight; for men, the sensitivity and specificity of BMI-specific WC cutoffs was estimated to be 0.74 and 0.55 , respectively, compared with 0.40 and 0.81 , respectively, for the single WC cutoff; for women, the sensitivity and specificity of BMI-specific WC cutoffs was estimated to be 0.74 and 0.61 , respectively, compared with 0.56 and 0.82 , respectively, for the single WC cutoff. In those subjects with excess body weight, the BMI-specific WC cutoffs provided higher specificity and a more appropriate balance of sensitivity and specificity than the single WC cutoff ( 0.62 and 0.54 vs. 0.95 and 0.11 , respectively, for men and 0.66 and 0.53 vs. 0.98 and 0.07 , respectively, for women).

\section{Discussion}

A number of studies have been conducted on the association between WC and clustering of cardiovascular risk factors. However, there have been few attempts to compare the association between WC and clustering of cardiovascular risk factors across BMI categories. Using the 2008 health 
Table 3 Sensitivity and specificity of single and BMI-specific waist circumference cutoffs to discriminate subjects with and without a clustering of cardiovascular risk factors

\begin{tabular}{|c|c|c|c|c|c|c|c|c|}
\hline \multirow[t]{2}{*}{ BMI } & \multicolumn{4}{|l|}{ Men } & \multicolumn{4}{|l|}{ Women } \\
\hline & $\begin{array}{l}\text { Cutoff } \\
(\mathrm{cm})\end{array}$ & $\begin{array}{l}\text { Prevalence of high } \\
\text { waist circumference }(\%)\end{array}$ & Sensitivity & Specificity & $\begin{array}{l}\text { Cutoff } \\
(\mathrm{cm})\end{array}$ & $\begin{array}{l}\text { Prevalence of high } \\
\text { waist circumference }(\%)\end{array}$ & Sensitivity & Specificity \\
\hline \multicolumn{9}{|c|}{ International Diabetes Federation cutoffs } \\
\hline All & 90 & 21.2 & 0.46 & 0.83 & 80 & 31.2 & 0.81 & 0.71 \\
\hline Underweight & 90 & 0.1 & 0.00 & 1.00 & 80 & 0.2 & 0.00 & 1.00 \\
\hline Normal weight & 90 & 4.2 & 0.10 & 0.97 & 80 & 26.9 & 0.64 & 0.74 \\
\hline Overweight & 90 & 63.9 & 0.74 & 0.41 & 80 & 95.5 & 0.98 & 0.05 \\
\hline \multicolumn{9}{|c|}{ Japanese Committee cutoffs } \\
\hline All & 85 & 40.9 & 0.71 & 0.65 & 90 & 8.3 & 0.44 & 0.93 \\
\hline Underweight & 85 & 0.2 & 0.00 & 1.00 & 90 & 0.0 & 0.00 & 1.00 \\
\hline Normal weight & 85 & 21.7 & 0.41 & 0.80 & 90 & 2.0 & 0.12 & 0.98 \\
\hline Overweight & 85 & 90.9 & 0.95 & 0.11 & 90 & 54.8 & 0.70 & 0.48 \\
\hline \multicolumn{9}{|c|}{ BMI-specific cutoffs } \\
\hline All & 85 & 40.9 & 0.71 & 0.65 & 81 & 28.1 & 0.78 & 0.74 \\
\hline Underweight & 69 & 47.2 & 0.76 & 0.54 & 67 & 49.9 & 0.70 & 0.50 \\
\hline Normal weight & 81 & 47.7 & 0.74 & 0.55 & 78 & 37.3 & 0.74 & 0.64 \\
\hline Overweight & 92 & 50.6 & 0.62 & 0.54 & 91 & 49.4 & 0.66 & 0.53 \\
\hline
\end{tabular}

$B M I$ body mass index

examination data of a Japanese health service association, we have been able to demonstrate that high WC is associated with an increased risk of clustering of cardiovascular risk factors independent of BMI. In our study, both the magnitude of the association and the ability of WC to discriminate subjects with and without a clustering of cardiovascular risk factors varied with obesity (BMI) status.

The multiple logistic regression analysis revealed a significant association between WC and clustering of cardiovascular risk factors in all BMI groups, with the exception of the underweight group of women. The ROC analysis showed a moderate ability of WC to discriminate subjects with and without a clustering of cardiovascular risk factors in all BMI groups. These results reinforce the importance of the measurement of WC in addition to BMI for cardiovascular risk assessment [16]. Although BMI and WC are highly correlated, they measure different aspects of obesity; WC, in contrast to BMI, can provide information on fat distribution [17]. A combination of BMI and WC may be a better risk measure than BMI alone.

The magnitude of the association between WC and clustering of cardiovascular risk factors, as indicated by the adjusted OR, was smaller in the overweight group than in the normal weight and underweight groups. This result does not refute the beneficial effect of WC reduction on cardiovascular risk factors in people with excess weight. However, the same 5-cm difference in WC may have a smaller impact on subjects with excess weight than on subjects without excess weight. In line with the difference in the magnitude of the association between WC and clustering of cardiovascular risk factors, the ability of WC to discriminate subjects with and without a clustering of cardiovascular risk factors, as indicated by the AUC, was smaller in the overweight group than in the normal weight and underweight groups. The measurement of WC is simple, inexpensive, and noninvasive, which may be suitable for the first step of cardiovascular risk assessment in the general population. Overall, the findings of this study support the introduction of WC to the nationwide strategy for detection of the metabolic syndrome [2]. However, health professionals should take notice that WC is likely to add limited information to that already provided by BMI when used for cardiovascular risk assessment in people with excess weight.

If $\mathrm{WC}$ is intended to be used for identifying people at an increased risk of developing cardiovascular disease, the most discriminative threshold of WC needs to be determined by the ROC analysis. When the single WC cutoffs recommended by the IDF [15] and by the Japanese Committee [3] were applied to the study subjects, the prevalence of high WC (percentage of subjects with a WC above the cut-off) differed widely between the BMI groups. As a consequence of the unbalanced prevalence of high WC, the single WC cutoffs recommended were less sensitive in the underweight and normal weight groups and less specific in the overweight group. The extremely low sensitivity in the underweight and normal weight groups means that the use of the single WC cutoffs recommended may lead to an 
insufficient identification of people without excess weight but with a clustering of cardiovascular risk factors.

The findings of this study support the conclusions drawn by Ardern et al. [18]. Using data from the National Health and Nutrition Examination Survey III and the Canada Heart Health Survey, these researchers revealed that the single WC cutoffs recommended by the National Institute of Health and the Health Canada $(102 \mathrm{~cm}$ for men and $88 \mathrm{~cm}$ for women) were inadequate to discriminate cardiovascular risk within all BMI categories. Moreover, they developed original BMI-specific WC cutoffs and confirmed that the identification of people at increased risk of developing cardiovascular disease was improved by using the BMI-specific WC cutoffs.

According to the results of the ROC analysis in each BMI group, the most discriminative threshold of WC showed an approximately $10 \mathrm{~cm}$ difference between the groups. The BMI-specific WC cutoffs provided a more appropriate balance of sensitivity and specificity in all BMI groups, while the discriminatory ability of BMI-specific WC cutoffs in the total study group was lower than that of the single WC cutoffs obtained from this study. Only in the subjects without excess weight (the combined underweight and normal weight group), did the BMI-specific WC cutoffs provide a substantial improvement in sensitivity. Further studies are necessary to confirm these findings in other populations. However, it is worth noting that the BMI-specific WC cutoffs may have a potential for better identification of people without excess weight but with a clustering of cardiovascular risk factors. Based on the results of the 2008 National Health and Nutrition Survey, $71.4 \%$ of men and $79.4 \%$ of women aged $\geq 20$ years are estimated to have a BMI $<25.0$ in Japan [1]. The NIPPON DATA Research Group revealed that the prevalence of people without excess weight but with a clustering of cardiovascular risk factors is quite high and that their cardiovascular mortality risk is also high [19]. A better identification of people without excess weight but with a clustering of cardiovascular risk factors would seem to be a valuable step in terms of improving public health in Japan.

The findings of this study are insufficient to recommend the combined BMI-WC screening. Further studies are necessary to validate the BMI-specific WC cutoffs using representative samples of the general population. The discriminatory ability of BMI-specific WC cutoffs, compared with the existing (recommended) WC cutoffs, should be confirmed in several different populations. On the other hand, health professionals should take notice that because of a low prevalence of clustering of cardiovascular risk factors, the number of false-positive cases, indicated by a 1 - positive predictive value (PPV), is substantial in people without excess weight. This misclassification is not prevented by using BMI-specific cutoff; the PPV of BMI- specific WC cutoffs was estimated to be 0.15 for men and 0.03 for women compared with the PVV of 0.19 and 0.05 , respectively, of the single WC cutoffs obtained in this study.

A main advantage of this study is the large number of study subjects with a wide age range. Our findings provide valuable information on the relationships among BMI, WC, and cardiovascular risk factors in Japanese adults. On the other hand, the study subjects consisted of participants in the 2008 health examination at a Japanese health service association; as such, they may not accurately represent the general population. Caution should be taken when generalizing the findings of this study. Moreover, the crosssectional design makes it difficult to determine the causal relationship between WC and clustering of cardiovascular risk factors. Miyatake et al. [20] reported that at least $3 \mathrm{~cm}$ of WC reduction may be beneficial for improving metabolic syndrome in obese Japanese men. Longitudinal studies may be required to address the question of to what extent is the increased risk of clustering of cardiovascular risk factors attributable to an increase in $\mathrm{WC}$ and to clarify the beneficial effect of WC reduction on cardiovascular risk factors in the context of potential BMI dependence.

\section{Conclusion}

Using the 2008 health examination data of a Japanese health service association, the results reported here demonstrate that, high WC was associated with an increased risk of clustering of cardiovascular risk factors independent of BMI. Both the magnitude of the association and the ability of WC to discriminate subjects with and without a clustering of cardiovascular risk factors varied with obesity (BMI) status. We suggest that the BMI-specific WC cutoffs have the potential to provide healthcare providers with a better tool for identifying people without excess weight but with a clustering of cardiovascular risk factors. Further studies are necessary to confirm the discriminatory ability of BMI-specific WC cutoffs before combined BMI-WC screening programs can be recommended.

Acknowledgments We are grateful to the staff of the Tokyo Health Service Association for data collection. The authors have received no financial support for this study.

Conflict of interest The authors declare that there is no conflict of interest.

\section{References}

1. Ministry of Health, Labour, and Welfare. The results of the 2008 National Health and Nutrition Survey (in Japanese). Available at: 
http://www.mhlw.go.jp/houdou/2009/11/h1109-1.html. Accessed 5 Feb 2010

2. Ministry of Health, Labour, and Welfare. Health examination and guidance program for Japanese adults (in Japanese). Available at: http://www.mhlw.go.jp/bunya/shakaihosho/iryouseido01/info02a. html. Accessed 5 Feb 2010

3. Examination Committee of Criteria for Metabolic Syndrome. Definition and criteria for metabolic syndrome in Japan (in Japanese). J Jpn Soc Intern Med. 2005;94:794-809.

4. Hara K, Matsushita Y, Horikoshi M, Yoshiike N, Yokoyama T, Tanaka H, Kadowaki T. A proposal for the cutoff point of waist circumference for the diagnosis of metabolic syndrome in the Japanese population. Diabetes Care. 2006;29:1123-4.

5. Lee JS, Kawakubo K, Mori K, Akabayashi A. Effective cut-off values of waist circumference to detect the clustering of cardiovascular risk factors of metabolic syndrome in Japanese men and women. Diabetes Vasc Dis Res. 2007;4:340-5.

6. Miyatake N, Wada J, Matsumoto S, Nishikawa H, Makino H, Numata T. Re-evaluation of waist circumference in metabolic syndrome: a comparison between Japanese men and women. Acta Med Okayama. 2007;61:167-9.

7. Nishimura R, Nakagami T, Tominaga M, Yoshiike N, Tajima N. Prevalence of metabolic syndrome and optimal waist circumference cut-off values in Japan. Diabetes Res Clin Pract. 2007;78:77-84.

8. Matoba Y, Inoguchi T, Nasu S, Suzuki S, Yanase T, Nawata H, Takayanagi R. Optimal cut points of waist circumference for the clinical diagnosis of metabolic syndrome in the Japanese population. Diabetes Care. 2008;31:590-2.

9. Narisawa S, Nakamura K, Kato K, Yamada K, Sasaki J, Yamamoto M. Appropriate waist circumference cutoff values for persons with multiple cardiovascular risk factors in Japan: a large cross-sectional study. J Epidemiol. 2008;18:37-42.

10. Shimajiri T, Imagawa M, Kokawa M, Konami T, Hara H, Kyoku I, Sone E, Ishigame M, Kikuoka H. Revised optimal cut-off point of waist circumference for the diagnosis of metabolic syndrome in Japanese women and the influence of height. J Atheroscler Thromb. 2008;15:94-9.

11. Oka R, Kobayashi J, Yagi K, Tanii H, Miyamoto S, Asano A, Hagishita T, Mori M, Moriuchi T, Kobayashi M, Katsuda S,
Kawashiri MA, Nohara A, Takeda Y, Mabuchi H, Yamagishi M. Reassessment of the cutoff values of waist circumference and visceral fat area for identifying Japanese subjects at risk for the metabolic syndrome. Diabetes Res Clin Pract. 2008;79: 474-81.

12. Takahashi M, Shimomura K, Proks P, Craig TJ, Negishi M, Akuzawa M, Hayashi R, Shimomura Y, Kobayashi I. A proposal of combined evaluation of waist circumference and BMI for the diagnosis of metabolic syndrome. Endocr J. 2009;56:1079-82.

13. Satoh H, Kishi R, Tsutsui H. Body mass index can similarly predict the presence of multiple cardiovascular risk factors in middle-aged Japanese subjects as waist circumference. Intern Med. 2010;49:977-82.

14. Suka M, Yoshida K, Kawai T, Aoki Y, Yamane N, Yamauchi K. Age- and sex-specific reference intervals for 10 health examination items: mega-data from a Japanese Health Service Association (in Japanese). Rinsho Byori. 2005;53:599-607.

15. International Diabetes Federation. IDF worldwide definition of the metabolic syndrome. Available at: http://www.idf.org/ metabolic_syndrome. Accessed 5 Feb 2010.

16. Canoy D. Distribution of body fat and risk of coronary heart disease in men and women. Curr Opin Cardiol. 2008;23:591-8.

17. Snijder MB, van Dam RM, Visser M, Seidell JC. What aspects of body fat are particularly hazardous and how do we measure them? Int J Epidemiol. 2006;35:83-92.

18. Ardern CI, Janssen I, Ross R, Katzmarzyk PT. Development of health-related waist circumference thresholds within BMI categories. Obes Res. 2004;12:1094-103.

19. Kadota A, Hozawa A, Okamura T, Kadowak T, Nakmaura K, Murakami Y, Hayakawa T, Kita Y, Okayama A, Nakamura Y, Kashiwagi A, Ueshima H. Relationship between metabolic risk factor clustering and cardiovascular mortality stratified by high blood glucose and obesity: NIPPON DATA90, 1990-2000. Diabetes Care. 2007;30:1533-8.

20. Miyatake N, Matsumoto S, Fujii M, Numata T. Reducing waist circumference by at least $3 \mathrm{~cm}$ is recommended for improving metabolic syndrome in obese Japanese men. Diabetes Res Clin Pract. 2008;79:191-5. 